\title{
Les transporteurs d'hexoses
}

Michèle Guerre-Millo

\section{ADRESSE ET TIRÉS À PART}

M. Guerre-Millo : directeur de recherche à l'Inserm. Inserm U. 177, Institut biomédical des Cordeliers, 15-21, rue de l'Ecole de Médecine, 75007 Paris, France.

Les transporteurs membranaires du glucose appartiennent à deux familles distinctes. Les SGLT, co-transporteurs $\mathrm{Na}^{+}$/glucose, réalisent un transport actif de glucose dans le rein et l'intestin ; les GLUT relaient la diffusion facilitée du glucose dans pratiquement toutes les cellules. On connaît deux isoformes de SGLT et six isoformes de GLUT qui s'expriment de façon spécifique dans les divers tissus et ont des propriétés cinétiques variées, appropriées aux caractéristiques du transport du glucose dans le tissu et la cellule considérés. Chacune des isoformes semble jouer un rôle distinct dans l'homéostasie du glucose, permettant un contrôle strict de la glycémie malgré des variations constantes d'apport et de consommation. Des anomalies de certaines isoformes ont été impliquées dans l'obésité ou certains diabètes.

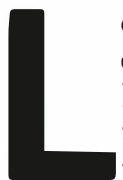

e glucose est un substrat énergétique majeur des cellules de mammifères. Pour traverser les membranes, cette petite molécule hydrosoluble est prise en charge par des protéines spécialisées, les transporteurs d'hexoses, certains d'entre eux étant capables de transporter également d'autres monosaccharides (galactose, mannose, fructose, etc.). Les transporteurs d'hexoses appartiennent à deux familles de protéines non apparentées: les SGLT (sodium-glucose transporters) assurent le transport actif et les GLUT (glucose transporters) la diffusion facilitée du glucose. Les SGLT comme les GLUT présentent des analogies avec des transporteurs de même type chez les organismes inférieurs, ce qui souligne la conservation du processus de transport des sucres au cours de l'évolution. Chez les mammifères, deux isoformes de SGLT et six isoformes de GLUT ont été identifiées à ce jour. L'apport le plus marquant des travaux de ces dix dernières années est la mise en évidence de la forte spécificité d'expression tissulaire des transporteurs, accompagnée de différences de propriétés cinétiques. La plupart des cellules expriment plusieurs isoformes qui "coopèrent" pour la réalisation optimale des fonctions mettant en jeu un transport d'hexose. Chaque isoforme paraît jouer un rôle particulier dans le maintien de l'homéostasie glucidique de l'organisme.

\section{La famille des SGLT}

Chez les mammifères, l'absorption et la réabsorption active du glucose ont lieu au niveau des cellules épithéliales de l'intestin grêle et du tube contourné proximal du néphron. Dans les deux cas, le transport du glucose est assuré par les transporteurs de la famille des SGLT. L'énergie nécessaire est fournie par le cotransport de $\mathrm{Na}^{+}$qui s'effectue dans le sens du gradient de concentration maintenu par la $\mathrm{Na}^{+} / \mathrm{K}^{+}$ 


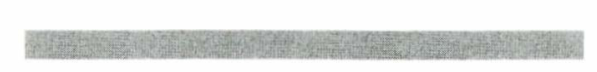

RÉFÉRENCES

1. Hediger MA, Coday MJ, Ikeda TS, Wright EM. Expression cloning and CDNA sequencing of the $\mathrm{Na}^{+}$/glucose co-transporter. Nature $1987 ; 330$ : 379-81

2. Turk E, Martin MG, Wright EM. Structure of the human $\mathrm{Na}^{+}$/glucose cotransporter. J Biol Chem 1994; 269 : 15204-9.

3. Burant CF, Flink S, DePaoli AM, Chen J, Lee WS, Hediger MA, Buse JB, Chang EB. Small intestine hexose transport in experimental diabetes. Increased transporter mRNA and protein expression in enterocytes. J Clin Invest 1994 ; 93: 578-85.

4. Kanai Y, Lee WS, You G, Brown D, Hediger MA. The human kidney low affinity $\mathrm{Na}^{+}$/glucose cotransporter SGLT2. Delineation of the major renal reabsorptive mechanism for D-glucose. J Clin Invest 1994; 93 : 397-404.

5. Mueckler M, Caruso C, Baldwin SA, Panico M, Blench I, Morris HR, Allard WJ, Lienhard GE, Lodish HF. Sequence and structure of a human glucose transporter. Science $1985 ; 229: 941-5$

6. Hresko RC, Kruse M, Srube M, Mueckler $M$. Topology of the Glut 1 glucose transporter deduced from glycosylation scanning mutagenesis. J Biol Chem 1994; 269: 204828.

7. Bell GI, Burant CF, Takeda J, Gould GW. Structure and function of mammalian facilitative sugar transporters. J Biol Chem 1993; 268: $19161-4$.

8. Oka Y, Asano T, Shibasaki Y, Lin JL, Tsukuda K, Katagiri H, Akanuma Y, Takaku $F$ C-terminal truncated glucose transporter is locked into an inward-facing form withou transport activity. Nalure 1990; 345: 550-3.

9. Mueckler M, Weng W, Kruse M. Glutamine 161 of Glut 1 glucose transporter is critical for transport activity and exofacial ligand binding. J Biol Chem 1994; 269 : 20533-8.

10. Mori $\mathrm{H}$, Hashiramoto M, Clark AE, Yang J, Muraoka A, Tamori Y, Kasuga M, Holman GD. Substitution of tyrosine 293 of GLUT1 locks the transporter into an outward facing conformation. J Biol Chem 1994; 269: 11578 83.

11. Pessin JE, Bell GI. Mammalian facilitative glucose transporter family: structure and molecular regulation. Annu Rev Physiol 1992; 54: 911-30.

\begin{tabular}{|c|c|c|c|c|}
\hline \multicolumn{5}{|c|}{$\begin{array}{c}\text { Tableau I } \\
\text { QUELQUES CARACTÉRISTIQUES DES TRANSPORTEURS D'HEXOSES }\end{array}$} \\
\hline Isoformes & $\begin{array}{l}\text { Nombre } \\
\text { d'acides } \\
\text { aminés }\end{array}$ & $\begin{array}{l}\text { Localisation } \\
\text { chromosomique } \\
\text { humaine }\end{array}$ & $\begin{array}{l}\text { Affinité } \\
\text { pour le glucose* } \\
\left(K_{m}\right)\end{array}$ & $\begin{array}{l}\text { Autres substrats } \\
\text { transportés }\end{array}$ \\
\hline $\begin{array}{l}\text { SGLT1 } \\
\text { SGLT2 }\end{array}$ & $\begin{array}{l}664 \\
672\end{array}$ & $\begin{array}{l}22 q 13.1 \\
16 p 11.2\end{array}$ & $\begin{array}{l}\text { Forte }(\approx 0,3 \mathrm{mM}) \\
\text { Faible }(\approx 2 \mathrm{mM})\end{array}$ & Galactose \\
\hline $\begin{array}{l}\text { GLUT1 } \\
\text { GLUT2 }\end{array}$ & $\begin{array}{l}492 \\
522\end{array}$ & $\begin{array}{l}1 p 35-31.3 \\
3 q 26\end{array}$ & $\begin{array}{l}\text { Forte }(\approx 7 \mathrm{mM}) \\
\text { Faible }(\approx 17 \mathrm{mM})\end{array}$ & $\begin{array}{l}\text { Galactose } \\
\text { Fructose, } \\
\text { galactose }\end{array}$ \\
\hline $\begin{array}{l}\text { GLUT3 } \\
\text { GLUT4 } \\
\text { GLUT5 } \\
\text { GLUT7 }\end{array}$ & $\begin{array}{l}596 \\
509 \\
501 \\
528\end{array}$ & $\begin{array}{l}12 \mathrm{p} 13 \\
17 \mathrm{p} 13 \\
1 \mathrm{p} 31 \\
?\end{array}$ & $\begin{array}{l}\text { Forte }(\approx 2 \mathrm{mM}) \\
\text { Forte }(\approx 5 \mathrm{mM}) \\
\text { Nulle } \\
?\end{array}$ & Galactose \\
\hline
\end{tabular}

* L'affinité pour le glucose des deux familles de transporteurs est différente, les SGLT ayant une plus forte affinité que les GLUT. Un transporteur est dit de faible ou de forte affinité en comparaison avec les transporteurs de sa propre famille.

ATPase présente sur la membrane basale des mêmes cellules.

\section{SGLT1, transporteur actif intestinal}

L'ADNc de la première isoforme de SGLT, SGLT1, a été obtenu en 1987 par Hediger et al. [1] grâce à une stratégie originale reposant sur la sélection de son ARNm par expression fonctionnelle d'ARNm d'intestin de lapin dans l'ovocyte de xénope. Des SGLTl très analogues ont été clonés ensuite dans plusieurs autres espèces. La séquence déduite de l'ADNc comporte 664 acides aminés chez l'homme (Tableau $I$ ) et s'insère dans la bicouche lipidique par 12 segments transmembranaires (figure 1) [2]. SGLTl a été localisé par immunohistochimie sur la bordure en brosse des cellules épithéliales de l'intestin grêle, ce qui est en accord avec son rôle présumé dans l'absorption du glucose alimentaire. La détection de deux mutations conduisant à une inactivation de SGLTl chez des patients atteints de malabsorption héréditaire de glucose/ galactose souligne l'importance physiologique de cette protéine [2]. La régulation de l'expression de SGLT1 commence à être étudiée. Ainsi, on a décrit chez le rat une augmentation de son expression dans l'intestin au cours du diabète insulinopénique expérimental, défaut qui se corrige lors du traitement par l'insuline [3].
SGLT2, transporteur actif du tube contourné proximal

L'utilisation de sondes dérivées de l'ADNc de SGLT1 a permis d'identifier chez l'homme une seconde isoforme SGLT2, produit d'un gène distinct, fortement exprimé dans le rein mais pas dans l'intestin. La caractérisation récente de SGLT2 a montré que cette isof orme a une affinité plus faible pour le glucose ('Tableau I) et une stœchiométrie différente, couplant le transport d'un $\mathrm{Na}^{+}$par glucose contre deux pour SGLT1 [4]. Les deux isoformes sont présentes dans le tube contourné, SGLT2 dans la partie proximale (segment S1) et SGLTl dans la partie distale (segment S3) [4]. L'existence de deux transporteurs présentant des caractéristiques cinétiques différentes est en accord avec la physiologie de la réabsorption du glucose dans le tube contourné. SGLT2 serait responsable de la réabsorption de $90 \%$ du glucose de l'urine primitive, SGLT1, ayant une plus forte affinité, assurant la réabsorption des dernières traces de glucose restant dans la partie distale. Les SGLT assurent l'entrée du glucose dans les cellules intestinales et rénales, d'où il doit sortir pour gagner le compartiment sanguin. Comme nous le verrons plus loin, ce processus a lieu par diffusion facilitée et est assuré par un GLUT (GLUT2). 


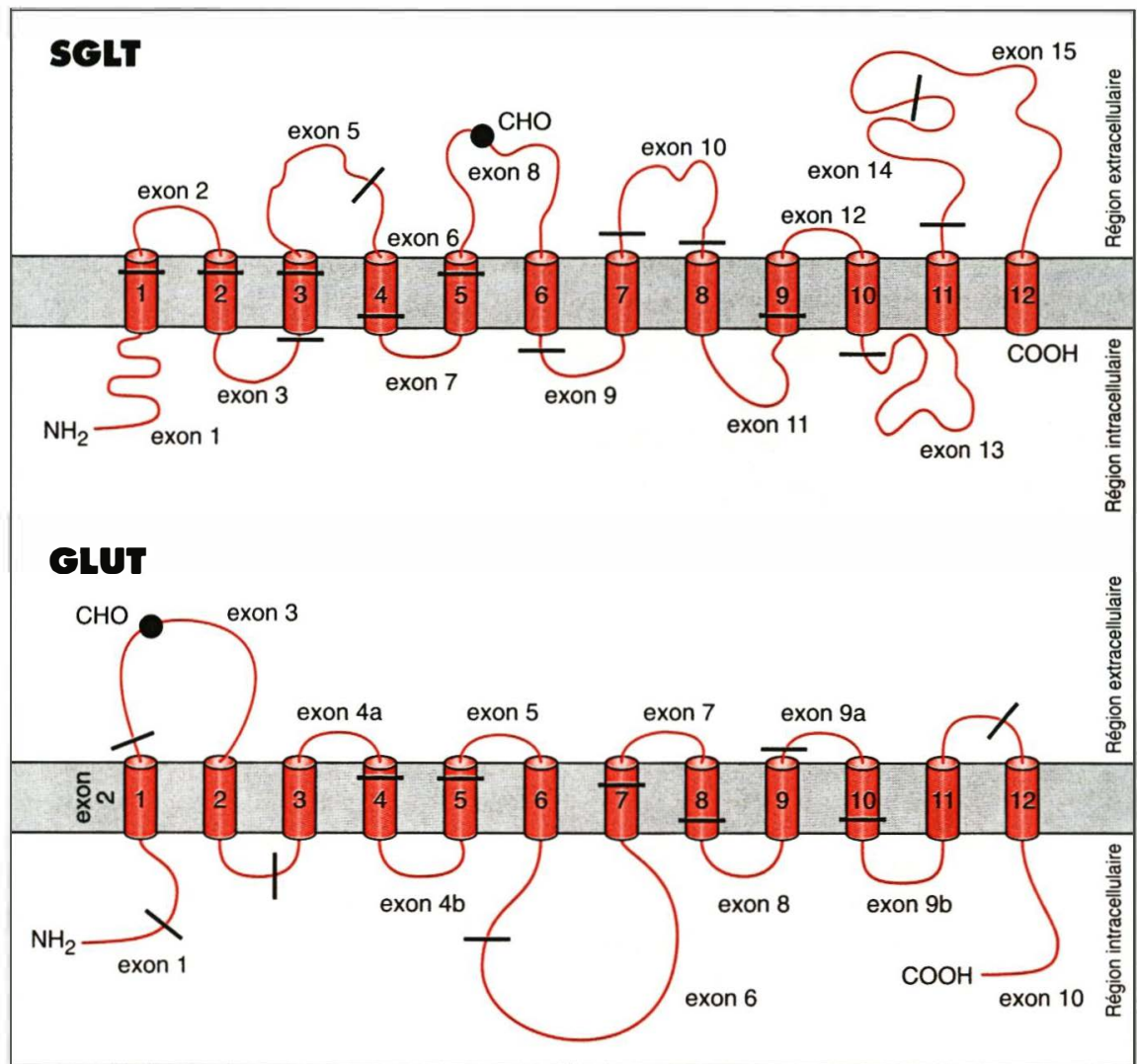

Figure 1. Modèle de structure des transporteurs d'hexoses déduit de leur séquence d'acides aminés. Les 12 segments transmembranaires sont représentés par des rectangles numérotés. $\mathrm{CHO}$ indique les sites de glycosylation Asn248 dans SGLT et Asn45 dans GLUT. Les extrémités $N$ - et C-terminales sont intracytoplasmiques. Les segments codés par les différents exons sont identifiés. (Adapté de [2] et [5].)

\section{La famille des GLUT}

GLUT1, modèle de structure et de fonctionnement

Le premier ADNc codant pour un GLUT, maintenant appelé GLUT1, a été isolé en 1985 par Mueckler et al. [5] à partir de cellules humaines d'hépatome (HepG2), en utilisant des anticorps dirigés contre le transporteur de l'érythrocyte humain partiellement purifié. L'analyse de la séquence déduite de l'ADNc de GLUT1 a conduit à proposer une structure secondaire comportant 12 segments transmembranaires organisés en hélice $\alpha$ (figure 1). Ce modèle a été confirmé par plusieurs études biophysiques et biochimiques et plus récemment par mutagenèse

8 et 11$)$ riches en acides aminés à groupes hydroxyle et amine [5]. Plusieurs études indiquent que GLUTl existe sous deux conformations présentant alternativement le site de liaison du glucose vers l'extérieur puis vers le cytoplasme [7]. Le glucose traverserait la membrane, dans un sens ou dans l'autre suivant le gradient de concentration, grâce au changement de conformation du transporteur qui, après libération du glucose, reprendrait sa conformation initiale. Le domaine C-terminal (37 acides aminés) semble être indispensable à ce cycle comme le montre l'analyse de GLUT1 tronqué de cette partie, qui reste bloqué avec le site de liaison vers le cytoplasme et est inactif [8]. $\mathrm{Au}$ contraire, le remplacement des acides aminés Gln-161 ou Tyr-293 dans les segments transmembranaires 5 et 7 bloque GLUT1 avec le site de liaison vers l'extérieur, ce qui conduit également à une réduction radicale du transport du glucose $[9,10]$. L'analyse détaillée du rôle des 492 acides aminés de GLUTl dans la constitution des sites de liaison du substrat et la fonction de transport est en cours. A ce jour, outre les remplacements cités précédemment, on sait que la mutagenèse dirigée de Trp412 et de Asn415 dans le segment transmembranaire 11 cause une inhibition quasi totale du transport du glucose et que la mutation du site de glycosylation Asn 45 modifie les propriétés cinétiques de GLUT 1, diminuant son affinité pour le substrat [7].

\section{Les isoformes de GLUT}

[6]. Bien que ne présentant pas d'analogie dans leur structure primaire, les GLUT et les SGLT ont une structure secondaire très comparable également partagée par d'autres types de transporteurs (transporteurs d'acides aminés, de nucléosides, etc.), qui pourrait donc être la plus efficace pour faire passer une molécule hydrosoluble à travers la bicouche lipidique.

Le mécanisme du transport du glucose à l'échelle moléculaire reste encore hypothétique et largement fondé sur le modèle de structure proposé pour GLUT1, du fait qu'il n'a pas été possible à ce jour de cristalliser un transporteur de glucose. GLUT1 fonctionnerait comme un canal hydrophile formé par 5 segments transmembranaires (hélices $\alpha 3,5,7$,
Il est rapidement apparu que le niveau d'expression de GLUT1 dans certains tissus gros consommateurs de glucose comme les muscles et le foie était relativement bas, d'où l'hypothèse de l'existence d'autres transporteurs. L'analyse systématique de banques d'ADNc de divers organes (foie, muscles, intestin) avec des sondes dérivées de GLUTl a permis d'isoler cing nouveaux ADNc numérotés GLUT2 à GLUT6 suivant l'ordre de leur découverte [11]. Ces ADNc ont tous été clonés dans plusieurs espèces, certaines isoformes étant très analogues (GLUT1, GLUT4) et d'autres plus divergentes (GLUT2, GLUT3). Un septième ADNc, GLUT7, a été 


\section{RÉFÉRENCES}

12. Waddell ID, Zomershoe AG, Voice MW Burchell $A$. Cloning and expression of a hepatic microsomal glucose transport protein. Comparison with liver plasma-membrane glucose-transport protein GLUT 2 Biochem J 1992; 286 : 173-7.

13. Maher F, Vannucci SJ, Simpson IA. Glucose transporter proteins in brain. FASEB 1994; 8 : 1003-11.

14. Santalucia $T$, Camps $M$, Castello $A$ Munoz P, Nuel A, Testar X, Palacin M, Zor zano $A$. Developmental regulation o GLUT-1 (erythroid/HepG2) and GLUT-4 (muscle/fat) glucose transporter expression in rat heart, skeletal muscle, and brown adipose tissue. Endocrinology 1992; 130: 837-46.

15. Bilir BM, Linda Gong TW, Kwasiborski V, Shen CS, Fillmore CS, Berkowitz CM Gumucio IJ. Novel control of the positiondependent expression of genes in hepato cytes. The GLUT-1 transporter. I Biol Chem $1993 ; 268: 19776-84$

16. Tal M, Kahn BB, Lodish HF. Expression of the low Km GLUT-1 glucose transporter is turned on in perivenous hepatocytes of insulin-deficient diabetic rats. Endocrinology $1991 ; 129: 1933-41$.

17. Wertheimer E, Sasson S, Cerasi E, BenNeriah Y. The ubiquitous glucose transporter GLUT-1 belongs to the glucose-regulated protein family of stress-inducible proteins. Proc Natl Acad Sci USA 1991; 88 2525-9.

18. Marshall BA, Ren JM, Johnson DW, Gibbs EM, Lillquist JS, Soeller WC, Holloszy JO, Mueckler M. Germline manipulation of glucose homeostasis via alteration of glucose transporter levels in skeletal muscle. $J$ Biol Chem 1993; 268: 18442-5.

19. Ren JM, Marshall AB, Gulve EA, Gao J, Johnson DW, Holloszy JO, Mueckler M. Evidence from transgenic mice that glucose transport is rate-limiting for glycogen deposition and glycolysis in skeletal muscle. / Biol Chem 1993; 268: 16113-5.

20. Simpson IA, Chundu KR, Davies-Hill T Honer WG, Davies P. Decreased concentration of GLUT1 and GLUT3 glucose transporters in the brains of patients with Alzheimer's disease. Ann Neurol 1994; 35: 546-51.

21. Assimacopoulos-Jeannet F, Jeanrenaud B. Contrôle du transport du glucose par l'insuline. médecine/sciences 1989 ; $5: 33-41$.

22. Slot JW, Gueze HJ, Gigengack S, Lienhard GE, James DE. Immunolocalization of the insulin regulatable glucose transporter in brown adipose tissue of the rat. J Cell Biol identifié, uniquement chez le rat pour l'instant, à l'aide d'anticorps dirigés contre le transporteur microsomique des hépatocytes [12]. La parenté génétique des différentes isoformes est présentée dans la figure 2 [7]. L'analyse des séquences déduites des $\mathrm{ADNc}$ indique que la taille des GLUT varie de 492 à 528 acides aminés ('T'ableau I) et qu'ils ont tous une structure identique à celle de GLUTI, sauf GLUT6, pseudogène non exprimé en transporteur fonctionnel. Les différents GLUT sont les produits de gènes distincts dont la localisation chromosomique est connue chez l'homme (Tableau I). L'interprétation du rôle spécifique de chaque isoforme commentée cidessous repose sur l'étude de leur distribution cellulaire et subcellulaire (figure 3) et de leurs propriétés cinétiques, en particulier l'affinité pour le substrat ('Tableau I).

- GLUT1, GLUT3 et GLUT4, transporteurs à forte affinité pour le glucose. Le transporteur GLUT1 est l'isoforme la plus répandue, détectée dans pratiquement tous les tissus et cellules de l'organisme bien qu'à des concentrations variables. Cette isoforme représente environ $5 \%$ des protéines membranaires de l'érythrocyte. Elle est abondante également dans les tissus embryonnaires et fœtaux ainsi que dans le placenta et les barrières hémato-tissulaires. Chez l'adulte, GLUTl est fortement exprimé dans le cerveau où il a été détecté par immunohistochimie au niveau de la barrière hémato-encéphalique et dans plusieurs types cellulaires [13]. Dans les muscles et les tissus adipeux, l'expression de GLUT1 diminue rapidement après la naissance, alors que, parallèlement, celle de GLUT4, isoforme spécifique des tissus insulino-sensibles (voir plus loin) augmente [14]. Dans le foie, seuls certains hépatocytes contigus aux veinules hépatiques expriment GLUTl [15] et cette expression peut s'étendre à d'autres hépatocytes en cas de jeûne prolongé ou de diabète [16]. L'expression de GLUT1 est également inductible dans les cellules qui normalement ne l'expriment pas ou peu (hépatocytes, adipocytes, cellules $\beta$ du pancréas) par la mise en culture ainsi que par l'immortalisation, suggérant que
GLUT1 pourrait appartenir à la famille des protéines dites de stress [17]. Récemment, la concentration musculaire de GLUTl a été artificiellement accrue chez la souris par transgenèse de l'ADNc du GLUT1 humain sous contrôle du promoteur de la chaîne légère de la myosine $[18,19]$. Chez les animaux transgéniques, le transport du glucose mesuré in vitro dans différents muscles est nettement augmenté ainsi que le contenu en lactate et en glycogène, ce qui indique que le transport est une étape limitante de l'utilisation du glucose par le muscle. De plus, les souris transgéniques sont légèrement hypoglycémiques et présentent une amélioration de la tolérance au glucose après charge orale. Ces observations démontrent que l'on peut modifier l'intensité du flux de glucose dans le muscle en manipulant le niveau d'expression de GLUTl et que cette opération a un effet sur l'homéostasie glucidique de l'organisme entier.

L'isoforme GLUT3 est présente principalement dans le cerveau chez la plupart des espèces étudiées. Cependant, chez l'homme, son ARNm a été détecté dans de nombreux tissus sans que l'on sache encore si la protéine est également présente. Des études récentes chez le rat ont montré que GLUT3 est présent spécifiquement dans les neurones où il est plus abondant que GLUT1 [13]. GLUT3 ayant le Kın le plus bas (Tableau I), sa présence pourrait favoriser les neurones par rapport aux cellules gliales (qui contiennent GLUTl), aux faibles concentrations extracellulaires de glucose qui prévalent dans le cerveau. La coexistence des deux isoformes, GLUT1 sur la barrière hémato-encéphalique et GLUT3, dont l'affinité est supérieure, dans les neurones permet théoriquement le transport vectoriel du glucose, du plasma vers les neurones. Une étude réalisée chez des patients atteints de maladie d'Alzheimer a montré qu'il existe une diminution de la concentration de GLUT3 dans certaines régions du cerveau présentant un déficit d'utilisation de glucose [20], ce qui est une première indication de l'importance physiologique de cette isoforme.

GLUT4 est présent dans les tissus où le transport du glucose est sensible à 


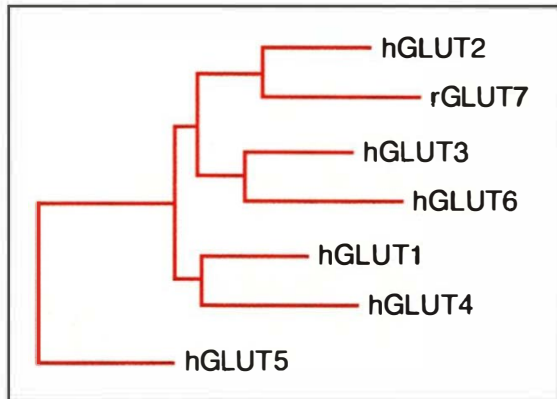

Figure 2. Représentation graphique des analogies entre les GLUT. $h$ : humain; r: rat. (D'après [7]). l'insuline, muscles et tissus adipeux [21]. Ce transporteur est étroitement impliqué dans l'action de l'hormone sur ses cellules cibles. Dans la cellule adipeuse, plus de $90 \%$ du GLUT4 est localisé sur des vésicules intracellulaires, le reste étant présent sur la membrane plasmique. En l'absence de l'hormone, le taux d'échange entre les deux compartiments est très faible. L'insuline, par liaison à son récepteur membranaire, engendre un signal qui déclenche très rapidement une accélération du mouve- ment des vésicules conduisant GLUT4 vers la membrane plasmique $\left(\mathrm{m} / \mathrm{s} n^{\circ} 10\right.$, vol. 10, p. 1051). Les transporteurs vont alors s'intégrer à la membrane plasmique après fusion des vésicules avec celle-ci, permettant l'entrée massive du glucose dans la cellule. Puis les GLUT4 regagnent l'intérieur de l'adipocyte, probablement par la voie des puits recouverts et des endosomes [22]. Ce phénomène décrit pour la première fois dans les années 1980 [23, 24], alors qu'on ignorait l'existence de plusieurs iso-
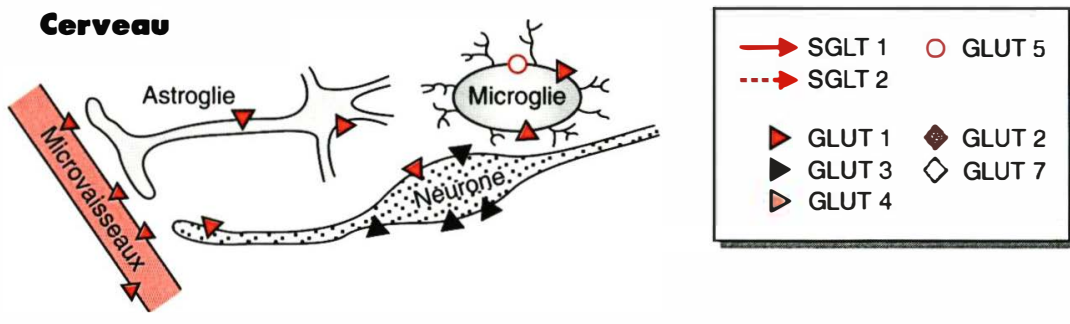

Intestin

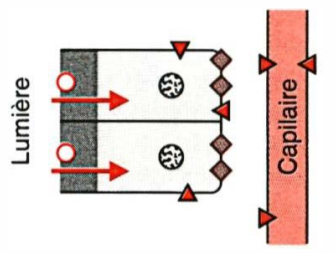

Rein

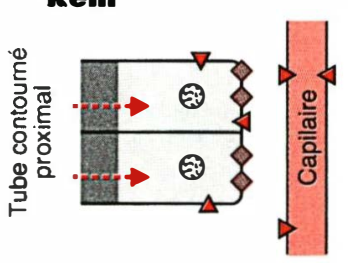

Pancréas endocrine

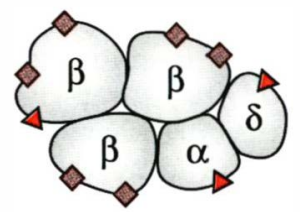

îlots $\beta$
Erythrocyte

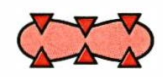

Foie

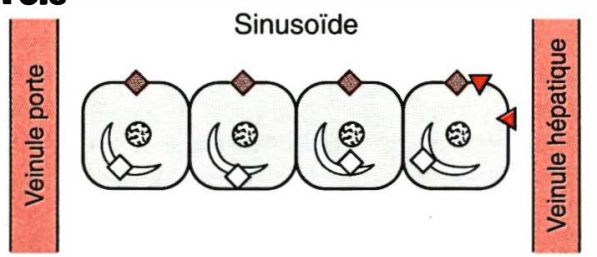

Tissus sensibles d̀ l'insuline

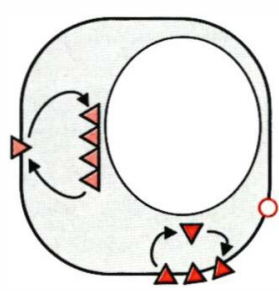

Cellule adipeuse

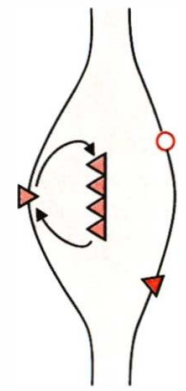

Cellule musculaire

Figure 3. Représentation schématique de la localisation cellulaire et subcellulaire des transporteurs d'hexoses. Les différentes isoformes exprimées dans les tissus jouent des rôles dépendant de leurs propriétés cinétiques et de leur localisation subcellulaire. Les SGLT réalisent le transport actif du glucose au niveau de l'intestion et du rein. Les GLUT relaient la diffusion facilitée. GLUT1 est l'isoforme la plus répandue. GLUT3, transporteur qui a la plus forte affinité pour le glucose, favorise la fourniture de glucose aux neurones. GLUT4 est exprimé dans les tissus où le transport du glucose est sensible à l'insuline. Localisé à l'intérieur des cellules, GLUT4 gagne la membrane plasmique en réponse à l'insuline permettant ainsi l'entrée massive de glucose. Dans la cellule adipeuse GLUT 4 est largement majoritaire. GLUT 1 est aussi présent, essentiellement à la membrane plasmique; il est aussi recyclé à la membrane en présence $d^{\prime}$ insuline. La faible affinité de GLUT2 pour le glucose lui permet $d^{\prime}$ avoir une activité directement proportionnelle à la glycémie. On le trouve dans les hépatocytes, sur les membranes baso-latérales des cellules épithéliales de l'intestin grêle et du tube contourné proximal du néphron où il prend le relais des SGLT en relarguant dans la circulation le glucose absorbé activement et dans les cellules $\beta$ du pancréas où il participe probablement à la signalisation de l'insulino-sécrétion. GLUT5, isoforme la plus divergente de la famille est un transporteur de fructose. Enfin, GLUT7, moins bien caractérisé, ferait partie du complexe enzymatique glucose-6-phosphatase des hépatocytes. 


\section{RÉFÉRENCES}

23. Cushman SW, Wardzala, IJ. Potential mechanism of insulin action on glucose transport in the isolated rat adipose cells. $J$ Biol Chem 1980; 255: 4755-62.

24. Suzuki K, Kono T. Evidence that insulin causes translocation of glucose transport activity to the plasma membrane from an intracellular storage site. Proc Nall Acad Sa USA $1980 ; 774: 2542-5$.

25. Holman GD, Kozka IJ, Clark AE, Flower C., Saltis I, Habberfield AD, Simpson IA Cushman SW. Cell surface labeling of glucose transporter isoform GLUT4 by bismulation of glucose transport in rat adipose cell by insulin and phorbol ester. J Biol Chem $1990 ; 265: 18172-9$

26. Wilson CM, Cushman SW. Insulin stimulation of glucose transport activity in rat skeletal muscle: increase in cell surface GLUT4 as assessed by photolabelling. Bio chem J 1994; 299: 755-9.

97. Satoh $\mathrm{S}$, Nishimura $\mathrm{H}$, Clark AE, Kozka I], Vannucci SJ, Simpson IA, Quon MJ Cishman SW, Holman CD. Use of bis-mannose photolabel to elucidate insulin-regulated GLUT4 subcellular trafficking kinetics in rat adipose cells. Evidence that exocytosis is a critical site of hormone action. $\int \mathrm{Biol}$ Chem 1993; 268 : 17820-9.

28. Verhey KJ, Birnbaum MI. A Leu-Leu sequence is essential for $\mathrm{COOH}$-terminal targeting signal of GLUT4 glucose transporter in fibroblasts. J Biol Chem 1994; 69 : 235.3-6.

29. Mastick CC, Aebersold R, Lienhard GE. Characterization of a major protein in GLUT4 vesicles. Concentration in the vesicles and insulin-stimulated translocation to the plasma membrane. I Biol Chem 1994; 269: 6089-92

30. Kandror KV, Pilch PF. gpl60, a tissuespecific marker for insulin-activated glucose transport. Proc Natl Acad Sa USA 1994; 91 : 8017-21.

31. Cormont M, Tanti JF, Zahraoui A, Van Obberghen E, Tavitian A, Le MarchandBruste $Y$. Insulin and okadaic acid induce Rab4 redistribution in adipocytes. $J$ Biol Chem $1993 ; 268: 19110-7$

32. Kahn BB. Facilitative glucose transporters: regulatory mechanisms and dysregulation in diabetes.J Clin Invest 1992; 89: 1367-74

33. Granner DK, O'Brien RM. Molecular physiology and genetics of NIDDM. Diabetes
34. Hainault I, Guerre-Millo M, Guichard C, Lavau M. Differential regulation of adipose tissue glucose transporters in genetic obesity (fatty rat). Selective increase in the adipose cell/muscle glucose transporter (GLUT4) expression. J Clin Invest 1991; 87 : 1127-31.

35. Liu ML, Gibbs EM, McCoid SC, Milici A], Stukenbrok HA, McPherson RK, Treadway JL, Pessin JE. Transgenic mice expressing the human GLUT4/muscle-fat facilitative glucose transporter protein exhibit efficient glycemic control. Pror Nall Arad Sir USA 1993; 90 : 11346-50.

36. Shepherd PR, Gnudi L, Tozzo E, Yang $H$, Leach F, Kahn BB. Adipose cells hyperplasia and enhanced glucose disposal in transgenic mice overexpressing GLUT4 selectively in adipose tissue. I Biol chem 1993 ; $268: 22243-6$.

37. Hajduch EJ, Guerre-Millo MC, Hainault IA, Guichard CM, Lavau MM. Expression of glucose transporters (GLUTl and GL.UT4) in primary cultured rat adipocytes: differential evolution with time and chronic insulin effect. J Cell Biochem 1992; 49:251-8.

38. Marshall BA, Mueckler M. Differential effects of GLUT-1 or GLUT 4 overexpression on insulin responsiveness in transgenic mice. Am J Physiol 1994; 267 : E738-44.

39. Katagiri $H$, Asano $T$, Ishihara $H$, Tsukuda $\mathrm{K}$, Lin JL Inukai $\mathrm{K}$, Kikuchi $\mathrm{M}$, Yazaki $\mathrm{Y}$ Oka Y. Replacement of intracellular C-ter minal domain of GLUTl glucose transporter with that of GLUT2 increases $V_{\text {mux }}$ and $\mathrm{K}_{m}$ of transport activity. J Biol Chem 1992; 267: 22550-5.

40. Thorens B, Cheng ZQ, Brown D, Lodisl $\mathrm{HF}$. Liver glucose transporter: a basolateral protein in hepatocytes and intestine and kidney cells. Am J Physiol 1990; 259: C279 85.

41. Froguel P, Vionnet N, Gauguier D Vaxillaire M, Zouali H, Passa P, Velho G. Génétique du diabète non insulinodépendant. médecine/sciences 1994; 10: 795-804

42. Newgard CB. Cellular engineering and gene therapy strategies for insulin replacement in diabetes. Diabetes $1994 ; 43: 341-50$.

43. Valera A, Solanes G, Fernandez-Alvarez J. Pujol A, Ferrer I Asins G, Gomis R, Bosh Expression of GLUT-2 antisense RNA in $\beta$ cells of transgenic mice leads to diabetes. $J$ Biol Chem 1994; 269: 28543-6. formes de transporteurs, est largement confirmé aujourd'hui, et l'on sait que c'est l'apparition de l'isoforme GLUT4 sur la membrane qui rend compte de l'effet de l'insuline sur le transport du glucose dans l'adipocyte et le muscle squelettique [22, $25,26]$. Il a, en outre, été démontré que l'effet de l'insuline consiste essentiellement en une stimulation de "l'exocytose" de GLUT4, sans modification notable de la vitesse d'endocytose [27].

Les mécanismes moléculaires qui régissent les mouvements de GLUT4 reposent sur sa faculté d'être efficacement séquestré à l'intérieur des cellules. Cette propriété est une caractéristique de l'isoforme, comme l'ont montré les expériences d'expression ectopique dans des lignées où GLUT4 est toujours détecté à l'intérieur des cellules transfectées. En comparaison, GLUTl, en dépit de sa forte analogie avec GLUT4, est surtout localisé sur la membrane plasmique. Plusieurs équipes ont réalisé des chimères entre les deux isoformes pour préciser le ou les domaines de GLUT4 nécessaires à sa rétention intracellulaire. La majorité des auteurs s'accorde à penser que la partie C-terminale de GLUT4 est un déterminant majeur de sa localisation intracellulaire. Ce domaine contient un motif di-leucine dont l'importance a été démontrée par mutagenèse et qui est absent de la région C-terminale de GLUT1 [28].

Par ailleurs, il faut noter que l'expression de GL.UT4 par transfection dans des cellules non insulinosensibles ne confère pas la réponse à l'insuline du transport du glucose, même en présence d'un nombre suffisant de récepteurs. Cela peut s'expliquer par la nécessité d'un environnement cellulaire particulier pour permettre la migration des vésicules portant GLUT4 vers la membrane. Dans une première approche de caractérisation des facteurs nécessaires à cette fonction, plusieurs équipes ont montré que des protéines potentiellement impliquées dans le trafic vésiculaire sont présentes sur les vésicules intracellulaires portant GLUT4 dans l'adipocyte de rat (Tableau II). En outre, certaines de ces protéines subissent une délocalisation en réponse à 
Tableau II

PROTÉINES CO-LOCALISÉES AVEC GLUT4 SUR LES VÉSICULES INTRACELLULAIRES DANS LES ADIPOCYTES DE RAT

\begin{tabular}{|c|c|c|c|}
\hline Protéines & $\begin{array}{l}\text { Localisation } \\
\text { dans d'autres } \\
\text { cellules }\end{array}$ & $\begin{array}{l}\text { Délocalisation } \\
\text { en réponse } \\
\text { à l'insuline }\end{array}$ & Rèf. \\
\hline $\begin{array}{l}\text { Phosphatidylinositol } \\
\text { 4-kinase }\end{array}$ & Divers types de vésicules & & [52] \\
\hline VAMP* & Vésicules synaptiques & Membrane plasmique & [53] \\
\hline SCAMP** & $\begin{array}{l}\text { Vésicules de sécrétion } \\
\text { et d'endocytose }\end{array}$ & Membrane plasmique & [54] \\
\hline $\begin{array}{l}\text { Rab4 } \\
\text { vp165, gp160 }\end{array}$ & Endosomes précoces & $\begin{array}{l}\text { Cytoplasme } \\
\text { Membrane plasmique }\end{array}$ & $\begin{array}{l}{[31]} \\
{[29,30]}\end{array}$ \\
\hline
\end{tabular}

* VAMP: vesicle-associated membrane proteins; * SCAMP: secretory carrier membrane proteins.

l'insuline, ce qui suggère leur participation aux mouvements de GLUT4. C'est grâce à ces propriétés, colocalisation avec GLUT4 et migration vers la membrane en réponse à l'insuline, que l'on a découvert une nouvelle protéine (vp165 ou gpl60), qui possède de surcroît la caractéristique de n'être exprimée que dans les tissus insulino-sensibles $[29,30]$. La fonction propre et l'implication de ces divers partenaires dans le trafic des vésicules portant GLUT4 restent encore largement hypothétiques. L'expression ubiquiste de certains suggère qu'ils participent probablement aux mécanismes généraux mis en jeu, alors que d'autres, comme gpl60, pourraient conférer la spécificité du processus aux cellules insulino-sensibles. L'hypothèse que la phosphorylation de la petite protéine $\mathrm{G}$ Rab 4 en réponse à l'insuline puisse être l'un des mécanismes impliqués a été récemment formulée par une équipe française [31]. La compréhension du rôle des différents acteurs dans le ciblage, la fusion avec la membrane plasmique et le recyclage des vésicules portant GLUT4 devrait permettre d'élargir notre connaissance des bases moléculaires de la sensibilité à l'insuline du transport du glucose.

Certaines situations pathologiques sont associées à une diminution de l'effet de l'insuline sur le transport du glucose dans les tissus cibles périphériques, ce qui détermine un état de résistance à l'hormone. C'est le cas du diabète non insulinodépendant (DNID). Plusieurs études s'accordent à montrer que l'expression de GLUT4 est notablement diminuée dans le tissu adipeux des patients DNID [32], ce qui peut rendre compte du défaut de transport du glucose. Cependant, de manière surprenante, GLUT4 n'est pas ou peu modifié dans les muscles squelettiques. Le défaut au niveau du muscle pourrait se situer "en aval" de l'étape transport, avec diminution de l'activité de l'hexokinase de type II, comme le suggèrent certains auteurs [33].

Par ailleurs, le rôle de GLUT4 dans le développement de l'obésité a été récemment mis en évidence. Nos travaux sur le rat Zucker génétiquement obèse ont montré que les adipocytes des jeunes rats mutants transportent considérablement plus de glucose que ceux des témoins et que cette surcapacité de transport repose entièrement sur une surexpression de GLUT4 [34]. Plus récemment, des souris transgéniques surexprimant GLUT4 dans le tissu adipeux ont été obtenues par transfection du gène humain, soit sous contrôle de son propre promoteur [35], soit sous contrôle du promoteur de la protéine aP2, spécifique du tissu adipeux [36]. Ces animaux présentent un accroissement important de la masse adipeuse, ce qui renforce l'idée que l'augmentation du flux de glucose dans les cellules adipeuses par surexpression de GLUT 4 conduit à l'obésité.

Les facteurs qui règlent la concentration de cette isof orme restent encore mal définis. Chez l'animal, les états d'insulinopénie comme le diabète expérimental ou le jeûne prolongé, s'accompagnent d'une forte diminution de GLUT4 dans le tissu adipeux, défaut corrigé par traitement à l'insuline ou renutrition [32]. Ces observations suggèrent que l'insuline pourrait jouer un rôle régulateur à long terme de l'expression adipocytaire de GLUT 4. Cependant, les études menées in vitro sur adipocytes en culture n'ont pas mis en évidence d'effet direct de l'insuline, suggérant l'intervention d'autres facteurs [37]. Dans le muscle squelettique, l'expression de GLUT4 est beaucoup plus stable que dans le tissu adipeux face aux mêmes perturbations métaboliques ou hormonales [32]. La base moléculaire de cette spécificité tissulaire de régulation est encore indéterminée. Une situation physiologique est cependant connue pour augmenter l'expression de GLUT4 dans le muscle squelettique : l'exercice physique prolongé [32]. L'observation de souris transgéniques surexprimant GLUT4 dans les muscles [38], qui présentent une augmentation de l'utilisation de glucose au niveau de l'organisme entier même sous hyperinsulinémie provoquée, suggère que l'augmentation du GLUT4 musculaire pourrait être un facteur d'amélioration de l'insulinorésistance.

- GLUT2, transporteur à faible affinité pour le glucose. L'isoforme GLUT2 se distingue des autres membres de la famille des GLUT par sa faible affinité pour le glucose (Tableau I). De ce fait, GLUT2 transporte le glucose proportionnellement à sa concentration, même aux valeurs élevées de glycémie ou de concentration intracellulaire. Cette propriété semble lui être conférée en grande partie par son domaine C-terminal. En effet, le transporteur chimérique GLUTl dont la partie C-terminale a été remplacée par celle de GLUT2 présente des caractéristiques cinétiques proches de celles de GLUT2 [39]. La répartition tissulaire de cette isoforme est limitée aux hépatocytes, aux cellules épithéliales de l'intestin grêle et du tube contourné proximal du néphron et aux cellules $\beta$ du pancréas. Dans l'intestin grêle et le néphron, GLUT2 a été localisé sur les membranes baso-laté- 


\section{RÉFÉRENCES}

44. Inman LR, McAllister CT, Chen L, Hughes S, Newgard CB, Kettman JR, Unger RH, Johnson JH. Autoantibodies to the GLUT-2 glucose transporter of $\beta$ cells in insulin-dependent diabetes mellitus of recent onset. Proc Natl Acad Sci USA 1993 ; $90: 1281-4$.

45. Mueckler M, Kruse M, Srube M, Riggs AC, Chiu KC, Permutt MA. A mutation in the GLUT 2 glucose transporter gene of a diabetic patient abolishes transport activity. J Biol Chem 1994; 269: 17765-7.

46. Tal M, Wu YJ, Leiser M, Surana M, Lodish $H$, Fleicher $N$, Weir $G$, Efrat $S$. (Val ${ }^{12}$ ) HRAS downregulates GLUT2 in $\beta$ cells of transgenic mice without affecting glucose homeostasis. Proc Natl Acad Sci USA 1992; 89: 5744-8.

47. Leloup C, Arluison M, Lepetit N, Cartier $N$, Marfaing-Jallat $P$, Ferré $P$, Pénicaud $L$. Glucose transporter 2 (GLUT 2): expression in specific brain nuclei. Brain Res 1994 ; sion in specific
$638: 221-6$.

48. Waeber $\mathrm{G}$, Thompson $\mathrm{N}$, Haefliger JA, Nicod $P$. Characterization of the murine high $K_{11}$ glucose transporter GLUT2 gene and its transcriptional regulation by glucose in a differentiated insulin-secreting cell line. J Biol Chem 1994; 269 : $26912-9$.

49. Lei KJ, Shelly LL, Lin B, Sidbury JB, Chen YT, Nordlie RC, Chou JY. Mutation in the glucose-6-phosphatase gene are associated with glycogen storage disease types la and laSP but not $1 \mathrm{~b}$ and $1 \mathrm{c}$. I Clin Invest $1995 ; 95$ : 234-40.

50. Burant CF, Takeda J, Brot-Laroche E Bell GI, Davidson NO. Fructose transporter in human spermatozoa and small intestine is GLUT5. J Biol Chem 1992; 267: 14523-6.

51. Shepherd PR, Gibbs EM, Wesslau C, Gould GW, Kahn BB. Human small intestine facilitative fructose/glucose transporter (GLUT5) is also present in insulin-responsive tissues and brain. Investigation of biochemical characteristics and translocation. Diabetes $1992 ; 41$ : 1360-5.

52. Del Vecchio RL, Pilch PF. Phosphatidylinositol 4-kinase is a component of glucose transporter (GLUT 4)-containing vesicles. $J$ Biol Chem 1991 ; 266 : 13278-83.

53. Cain CC, Trimble WS, Lienhard GE Members of the VAMP family of synaptic vesicle proteins are components of glucose transporter-containing vesicles from rat adipocytes. J Biol Chem 1992; 267 : 11681-4.

54. Laurie SM, Cain CC, Lienhard GE, Castle JD. The glucose transporter GLUT4 and secretory carrier membrane proteins (SCAMPs) colocalize in rat adipocytes and partially segregate during insulin stimula- rales [40], c'est-à-dire à l'opposé des transporteurs actifs SGLT. Cette localisation suggère que GLUT2 prend le relais des SGLT en transportant vers le sang le glucose absorbé activement au niveau des membranes apicales. Dans les hépatocytes, GLUT2 est également face au compartiment sanguin [40] où il assure la capture ou l'exportation du glucose, suivant l'état physiologique. Cette isoforme est probablement aussi responsable de la capture du fructose par le foie.

Dans les cellules $\beta$ du pancréas, ce sont les variations de la glycémie qui contrôlent la quantité d'insuline délivrée dans le sang, par un mécanisme fidèle et précis nécessitant à la fois le transport et le métabolisme du glucose. Le transporteur GLUT2 et la glucokinase, première enzyme de la glycolyse phosphorylant le glucose, sont potentiellement impliqués dans le système de signalisation de la réponse insulino-sécrétoire [41]. En effet, leur caractéristique commune de faible affinité pour le substrat permet la "traduction" des changements de glycémie en modification de flux métabolique de glucose. Cette hypothèse est étayée par un ensemble de travaux portant sur des cellules antéhypophysaires AtT20ins, exprimant naturellement une glucokinase de type pancréatique et GLUT1. Manipulées génétiquement pour synthétiser et sécréter l'insuline humaine, elles ne répondent cependant pas au glucose. Seule la transfection d'un ADNc codant pour GLUT2 instaure la réponse insulinosécrétoire, ce qui les rapproche des “cellules $\beta$ artificielles" [42]. L'importance relative des deux partenaires, GLUT2 et glucokinase, dans le dispositif glucosenseur des cellules $\beta$ et le maintien d'une glycémie normale, est actuellement l'objet de controverses. Plusieurs types d'observations sont en faveur d'un rôle de GLUT2 ( $\mathrm{m} / \mathrm{s} n^{\circ} 3$, vol. 7, p. 291). Une diminution importante de l'expression pancréatique de cette isoforme est systématiquement observée dans plusieurs modèles animaux de diabète, parallèlement à un défaut de sécrétion d'insuline (références dans [43]). Des souris trangéniques exprimant un ARNm antisens de GLUT2 sous contrôle du promoteur de l'insuline présentent une forte réduction de l'expression de GLUT2 dans les cellules $\beta$ qui s'accompagne d'une diminution de la sécrétion d'insuline et du développement d'un diabète noninsulino dépendant [43]. Chez l'homme, des auto-anticorps inhibiteurs de GLUT2 ont été trouvés chez des patients présentant un diabète d'apparition récente [44]. De plus, une mutation dans le gène produisant un GLUT2 inactif a été récemment détectée chez une patiente atteinte d'un diabète gestationnel [45]. Cependant, d'autres travaux tendent à minimiser l'importance de GLUT2, comme par exemple le fait qu'une réponse insulino-sécrétoire et une glycémie normale existent même en présence d'une réduction de l'expression de GLUT2 chez des souris transgéniques surexprimant l'oncogène Ras dans les cellules $\beta[41,46]$. Ces observations renforcent l'hypothèse selon laquelle la capacité de transport dépasserait largement la capacité de phosphorylation du glucose dans les cellules $\beta$ et que seule une réduction majeure de la concentration ou de l'activité de GLUT2 entraînerait une altération de la réponse au glucose. Enfin, il faut noter la présence de GLUT2 dans certains noyaux cérébraux dont l'activité varie avec la glycémie, ce qui suggère que GLUT2 pourrait également jouer un rôle glucosenseur au niveau du système nerveux central [47].

Les facteurs qui contrôlent l'expression de GLUT2 dans les cellules $\beta$ et, en particulier, ceux qui conduisent à sa diminution dans le diabète sont encore mal définis. Comme c'est le cas pour GLUT4, la régulation de GLUT2 présente une spécificité tissulaire dont la base moléculaire reste à découvrir. En effet, si GLUT2 est fortement diminué dans le pancréas du rat diabétique, sa concentration n'est pas ou peu modifiée dans ses autres sites d'expression, foie, rein, intestin (dans [48]).

- GLUT7, transporteur microsomique hépatique. GLUT7 a été découvert par une équipe anglaise [12] étudiant la glucose-6-phosphatase. Cette enzyme hépatocytaire catalyse la déphosphorylation du glucose-6phosphate provenant de la néoglucogenèse ou de la glycogénolyse. Le 
glucose est produit dans la lumière du réticulum endoplasmique et doit, avant d'être exporté vers le sang par GLUT2, traverser la membrane du réticulum pour gagner le cytoplasme. C'est là qu'interviendrait GLUT7. Cette isoforme, pratiquement identique à GLUT2, se distingue par son extrémité C-terminale qui comporte une séquence consensus de protéine du réticulum endoplasmique. Des travaux récents suggèrent que la glucose-6-phosphatase est un complexe multiprotéique comportant une unité catalytique, la glucose-6-phosphatase proprement dite et trois protéines de transport du substrat (glucose-6-phosphate) et des produits (phosphate et glucose). Il reste à déterminer si GLUT7 fait effectivement partie de ce système complexe. A notre connaissance, il n'existe pas de données révélant la présence de GLUT7 dans d'autres organes (rein, intestin) dans lesquels l'enzyme est exprimée. Plusieurs mutations ont été détectées dans le gène humain de la glucose-6phosphatase qui déterminent une glycogénose de type la $\left(\mathrm{m} / \mathrm{s} n^{\circ} 12\right.$, vol. 9 , p. 1424) [49]. Cependant, dans d'autres glycogénoses de même type, le gène est normal, ce qui suggère un défaut au niveau des protéines de transport et donc potentiellement dans le gène codant pour GLUT7.

- GLUT5, transporteur de fructose. L'ARNm de GLUT5 est fortement exprimé dans l'intestin grêle et, à un niveau moindre, dans les tissus insulino-sensibles, le cerveau et le rein. Dans les cellules intestinales, GLUT5 est présent essentiellement sur la face luminale. Le rôle d'un GLUT à ce niveau est resté mal compris sachant que le captage du glucose alimentaire est réalisée par SGLT1, jusqu'au moment où des études cinétiques ont montré que GLUT5 est en fait un transporteur de fructose [50]. Il faut noter d'ailleurs que cette isoforme est la plus divergente de la famille des GLUT (figure 2). En bon accord avec sa capacité de transporter le fructose, GLUT5 est présent sur la membrane des spermatozoïdes humains. En revanche, il n'est pas présent dans le foie. Dans le tissu adipeux, on sait que GLUT5 est principalement sur la membrane plasmique et ne subit pas de translocation en réponse à l'insuline, contrairement à GLUT4 (et à GLUT1) [51].

\section{Conclusions}

Le transport du glucose de l'extérieur de l'organisme vers le compartiment sanguin puis les cellules est un processus vital pour les organismes supérieurs. Il s'accompagne d'un contrôle strict de la glycémie, qui s'exerce dans un contexte de perturbations constantes dans l'apport (repas, jeûne) et la consommation de glucose (exercice physique, repos). La complexité de cette régulation se reflète au niveau du nombre et de la spécialisation des protéines impliquées. Chaque transporteur de glucose joue un rôle qui lui est propre, un défaut de l'un des partenaires pouvant perturber l'homéostasie glucidique de l'organisme entier. Une meilleure connaissance du fonctionnement et de la régulation des transporteurs d'hexoses devrait permettre à plus long terme des avancées thérapeutiques et préventives en particulier dans le domaine de maladies fréquentes et invalidantes comme le diabète et l'obésité

\section{Remerciements}

L'auteur tient à remercier tout particulièrement Bernard Hainque pour ses critiques constructives et ses suggestions nombreuses au cours de la préparation de cet article, ainsi que Marcelle Lavau, Jean Guerre et Armelle Leturque pour leurs conseils.

\section{Summary}

Hexoses transporters

Glucose is a major source of metabolic energy for mammalian cells. The transport of glucose in and out of cells is carried out by specialized membrane proteins, which belong to two distinct gene families. The $\mathrm{Na}^{+} /$glucose cotransporters (SGLT) actively transport glucose against its concentration gradient, a process which takes place in gut and kidney. The second class of glucose transporters (GLUT) mediates the facilitative diffusion occurring in virtually every cell. Currently, two SGLT (SGLTl and 2) and six GLUT (GLUT 1, 2, 3, 4, 5 and 7) isoforms have been characterized in mammals. The most significant observation to arise in the field is the realization that they are expressed in a tissue-specific fashion and exhibit different kinetic properties which fit with diversity of glucose transport characteristics. Most tissues and cells express several transporters, being likely to cooperate to ensure efficient and specialized transport function. Various pathologies like sugar malabsorption, diabetes, obesity are associated with alteration in certain glucose transporter isof orms, indicating that each plays a distinct role in whole-body glucose homeostasis. 